\title{
Downscaling, parameterization, decomposition, compression: a perspective from the multiresolution analysis
}

\author{
J.-I. Yano \\ CNRM-GAME, Météo-France and CNRS, URA 1357, Toulouse, France
}

Received: 27 February 2009 - Revised: 29 May 2010 - Accepted: 11 June 2010 - Published: 22 June 2010

\begin{abstract}
Geophysical models in general, and atmospheric models more specifically, are always limited in spatial resolutions. Due to this limitation, we face with two different needs. The first is a need for knowing (or "downscaling") more spatial details (e.g., precipitation distribution) than having model simulations for practical applications, such as hydrological modelling. The second is a need for "parameterizing" the subgrid-scale physical processes in order to represent the feedbacks of these processes on to the resolved scales (e.g., the convective heating rate).

The present article begins by remarking that it is essential to consider the downscaling and parametrization as an "inverse" of each other: downscaling seeks a detail of the subgrid-scale processes, then the parameterization seeks an integrated effect of the former into the resolved scales. A consideration on why those two closely-related operations are traditionally treated separately, gives insights of the fundamental limitations of the current downscalings and parameterizations.

The multiresolution analysis (such as those based on wavelet) provides an important conceptual framework for developing a unified formulation for the downscaling and parameterization. In the vocabulary of multiresolution analysis, these two operations may be considered as types of decompression and compression. A new type of a subgrid-scale representation scheme, NAM-SCA (nonhydrostatic anelastic model with segmentally-constant approximation), is introduced under this framework.
\end{abstract}

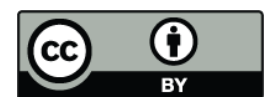

Correspondence to: J.-I. Yano (jun-ichi.yano@lmd.jussieu.fr)

\section{Introduction}

The present contribution intends to provide a particular perspective on representation of the subgrid-scale physical processes in geophysical modelling. Yano et al. (2005) have proposed that the subgrid-scale representation problem can systematically be considered as that of a mode decomposition. The present article generalizes this proposal by also including the downscaling within a perspective and also reports the author's more recent model development along this line.

Although the present article is written from an author's atmospheric perspective, he believes that it would equally be applicable to the ocean modellings, and to less extent, hydrological modellings. The geophysical system, in general, can be described by a set of partial differential equations. The atmosphere and ocean systems share much in common, adopting a system of equations derived as a certain approximation of the Navier-Stokes equation.

In standard procedures, these equations are solved numerically by using a discrete distribution of physical variables in space. For this reason, the original partial equations must be approximated, so that they are solvable in a discretized space. The simplest procedure is to approximate spatial partial differentiations by difference over two contiguous points, or finite differences. Due to a finite resolution in numerical modelling, physical processes that fall between the two continuous spatial points are no longer directly available. These physical processes that are not directly available are called subgrid-scale physical processes. These subgrid-scale processes must be somehow represented in an indirect manner, because they are otherwise not available. We call this problem subgrid-scale representation (SSR). 
SSR can be divided into two major parts. The first is the downscaling (e.g., Marani and Zanetti, 2007), a retrieval of details of the subgrid-scale processes, such as a spatial rainfall distribution of the scales finer than those explicitly resolved by a numerical weather forecast model. The second is the parameterization (cf., Arakawa, 2004), an estimation of the feedbacks of the subgrid-scale processes onto the resolved scales, such as the latent heating rate due to condensation of water vapor into liquid water associated with precipitation processes not spatially resolved by a numerical model. Note that the current weather forecasting models still have difficulties in resolving these precipitation processes in a manner that practical needs are well satisfied.

The present paper begins by making a relatively obvious point that these two types of SSR constitutes a pair problem in which the one can conceptually be considered as an "inverse" of the other. This point is made in the next section by presenting SSR in a general manner.

However, the current downscaling and parameterization schemes are not designed as such a pair operation. Thus, the two related questions follow: (1) Why the current downscaling and parameterization are not treated as an "inverse" of the other? (2) Then, how the downscaling and parameterization can be developed as a pair operation consistently? These two questions are addressed in Sect. 3 and 4, respectively. Section 5 presents a new type of SSR, called NAM-SCA (nonhydrostatic anelastic model with segmentally-constant approximation), as a specific application of the proposed principle. The paper is concluded in Sect. 6.

\section{The subgrid-scale representation problem}

An equation for any prognostic physical variable $\varphi$ (e.g., velocity, temperature, humidity, salinity, soil water) can be expressed by

$$
\frac{\partial}{\partial t} \varphi=-\frac{1}{\rho} \nabla \cdot \rho \boldsymbol{v} \varphi+F,
$$

where $\rho$ is the density, $\boldsymbol{v}$ is the velocity, and the last term $F$ represents all the other physical processes other than the advection that control the variable $\varphi$, which may be called "forcing" or "source".

A geophysical model is, however, usually solvable only numerically. A finite horizontal grid-box can be conceptually considered as a basic unit in the numerical computations: i.e., a model system is presented in terms of the grid-box averages. ${ }^{1}$ From this perspective, the subgrid-scale processes are what is going on within each model grid box.

\footnotetext{
${ }^{1}$ Strictly speaking, this statement is true only if the numerical code is written in terms of a finite volume. When a finite difference scheme is used, grid-box means of physical variables are not directly available, thus this argument is only conceptually correct.
}

A version of Eq. (1) averaged over a grid box is used in an actual numerical implementation:

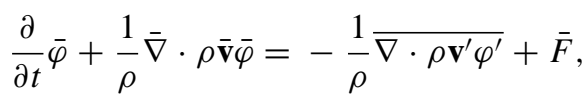

where the overbar designates the horizontal mean over the grid box, the prime indicates the deviation from this mean (e.g., $\varphi^{\prime}=\varphi-\bar{\varphi}$ ), and $\bar{\nabla}$ is a reminder that this nabla operation is performed over finite volume elements, or finite-distance grid points. In this derivation, we have assumed that the nabla operator $\nabla$ is interchangeable with the averaging operator, thus e.g., $\bar{\nabla} \bar{\varphi}=\bar{\nabla} \bar{\varphi}$.

The problem of SSR consists of defining the right-hand side of Eq. (2), which may be split in the two parts: firstly, subgrid-scale distributions $\varphi^{\prime}$ of variables must be defined. That constitutes the downscaling. Then the feedbacks of subgrid-scale variabilities, defined by the right-hand side above, are evaluated by these subgrid-scale variables obtained by downscaling. The second part constitutes the parameterization.

The evaluation of the grid-box averaged forcing $\bar{F}$ is generally more involved than the evaluation of the eddy fluxes given, as a first term in the right-hand side. Nevertheless, the main point is the same, that subgrid-scale distributions $\varphi^{\prime}$ of variables must be known before the forcing $\bar{F}$ can be evaluated.

Thus, the SSR problem consists of the two steps. First, the subgrid-scale variability, $\varphi^{\prime}$, is evaluated by downscaling. Second, the feedbacks of the subgrid-scale variabilities on the model-resolved scales (the right-hand side of Eq. 2) is, then, evaluated by parameterization. In this manner, the two problems, the downscaling and the parameterization constitute two parts of a single problem.

\section{Why the current downscaling and parameterization do not constitute two parts of a single problem?}

As far as the current state of art is concerned, the downscaling and the parameterization are never considered as a pair problem: why? Sociologically speaking, this situation may be attributed to an almost perfect separation of the two communities developing the downscaling and the parameterization without any link between them. Lack of communications also leads to inherent limitations of the current approaches both in downscaling and parameterization.

The main stream of downscalings (statistical downscaling) is usually performed in a purely statistical manner, with a focus on a physical variable of practical interests, more commonly the precipitation distribution (cf., Foufoula-Georgiou, 1997). No other physical variables, that are essential in estimating precipitation from a convection parameterization, are taken into account.

The current state of the art of the parameterization is equally limited. A particular example to make the point is 
the convection parameterization. A current main stream of the approach, called mass flux, is based on an idea of representing subgrid-scale convection by an ensemble of convective plumes, by taking mathematical elements called mass fluxes (Arakawa and Schubert, 1974). However, in the current standard approaches, no interaction between the plumes is considered, thus no geometrical information on a spatial distribution of plumes is taken into account. This is also generally the case with all the current existing parameterizations such as those based on the moment expansion, as well as on the probability distribution. In either case, by moving the problem into an abstract phase space, the geometrical information of the subgrid-scale processes is lost.

Downscaling is also often performed in a more direct manner, by a methodology more specifically called regionalization, in which a regional model is run locally over a particular region of an interest in order to numerically recover subgridscale details missing in a host model. Probably, the biggest irony may be found here: as a standard procedure, the regionalization is performed off-line only after a computation of the host model is completed. It is in spite of the fact that the regionalization provides the subgrid-scale information that can be directly used for evaluating their feedbacks into the resolved scales, if the two models were running in parallel. An interactive nesting approach may partially ${ }^{2}$ provide a remedy for this problem, but such an approach is rarely adopted by the downscaling community.

An opposite extreme situation is found in the so-called super-parameterization (Grabowski and Smolarkiewicz, 1999). This technique consists of placing an explicit model (the so-called cloud-resolving model) to each grid box of a global model. Limitations of the current approaches may be emphasized: the explicit model not only fails to fill a whole domain of the grid box, but it is also limited to two dimensions in geometry. Nevertheless, in spite of all these limitations, outputs from super-parameterization definitely provide information for downscaling, that has not been exploited in the author's best knowledge.

It would be relatively straightforward to place a full regional model or a cloud-resolving model into a few selected grid boxes, in the same spirit as regionalization, but under a fully interactive configuration as the super-parameterization. Thereinafter, downscaling and super-parameterization can be performed simultaneously. However, in the author's best knowledge, this attempt has not yet been made.

\section{How to construct the downscaling and parameterization together consistently?}

Then, how can we perform the downscaling and parameterization simultaneously together in a consistent manner? A hint for this answer is already provided in the last section:

\footnotetext{
${ }^{2}$ Unfortunately, the current standard nesting approaches provide a downscaling of only few factors.
}

if either a regional model or a cloud-resolving model can be placed at each grid box in such a way, that fills a full grid-box domain and fully interact with a host model, then the downscaling and super-parameterization can be performed simultaneously.

The purpose of SSR is to achieve the same result in a much more economic manner without calling for a full numerical model. Both the current statistical downscaling and parameterization are constructed under this philosophy. A way to obtain these two operations as a pair would be to reduce a full model into a compact description consistently. Importantly, the reduction must be performed in such a way, that the pair operation capacity in the full model is not lost as a result.

How such a reduction can be achieved? The image compression may provide a source of an inspiration. The basic idea of the image compression is to compress the size of an electronic image file without substantially deteriorating its quality. An easiest way of performing an image compression (cf., Mallat, 1998) is to represent an image, $\varphi(x, y)$, by wavelets, say,

$\chi_{l}(x, y) \quad(l=1, \ldots, N)$,

as

$\varphi(x, y)=\sum_{l=1}^{N} \tilde{\varphi}_{l} \chi_{l}(x, y)$.

Here $\tilde{\varphi}(l=1, \ldots, N)$ are the expansion coefficients. We retain only leading wavelet coefficients characterized by indices:

$l(i) \quad(i=1, \ldots, n)$

for representing this image with $n \ll N$. As a result, we obtain a compressed representation of the image:

$\varphi(x, y) \simeq \sum_{i=1}^{n} \tilde{\varphi}_{l(i)} \chi_{l(i)}(x, y)$.

By setting all the remaining less significant wavelet coefficients simply zero, the size of an image file can be substantially reduced, or compressed. In other words, a heavy truncation in wavelet space leads to an efficient image compression.

In fact, the file in concern is not necessarily an "image", but the same principle can be applied to any model outputs $\varphi(x, y, z, t)$ including the height, $z$ and the time, $t$ dependences. The applicability of the idea is not limited to such a static situation, but it can even be applied to a prognostic situation, i.e., in a context of time integration of a model itself. In other words, the time evolution of the wavelet coefficients $\tilde{\varphi}_{l(i)}(z, t)$ can be calculated in a compressed phase space with $i=1, \ldots, n$. Note that a prognostic set of equations for $\tilde{\varphi}_{l(i)}(z, t)$ is easily obtained by a transformed method. More extensive discussions on time integration methods are found in Yano et al. $(2004,2005)$ and the references therein. 
Once such an efficient compression of a full system is accomplished, say, in wavelet space, the SSR problem is effectively solved. The compressed output in the wavelet space can easily be used for evaluating the feedbacks to the resolved scales (i.e., the right-hand side of Eq. 2; cf., Yano et al., 2005), thus the parameterization is accomplished. Furthermore, once the compressed data are properly decompressed into a physical space, a full spatial distribution of a physical variable in concern is recovered, simply by evoking Eq. (4b), thus the downscaling is also accomplished.

The compression can be considered as a type of filtering, but here the filtering is performed in a highly nonlinear manner. Note that the truncation (Eq. 4a) is defined in much more general manner than any conventional filtering method. Characteristics of filtering is highly dependent on the position due to a spatially-localized nature of the wavelet. It may also be emphasized that the wavelet decomposition (Eq. 3a) considered here retains a spatial distribution of a physical variable. Unlike conventional parameterization approaches, a downscaling can be performed in a straightforward manner by Eq. (4b).

The proposed compression-decompression principle may be more generally stated as follows: (1) Identify the basic elements for given subgrid-scale physical processes. For atmospheric convection, these are traditionally considered as plumes (cf., Arakawa and Schubert, 1974; Fig. 1). (2) Perform a mode decomposition (Eq. 3b) of a given subgrid-scale system (e.g., a regional model, a cloud-resolving model). In order to ensure the efficiency of the mode decompression (so that a heavy truncation becomes possible), a set of mathematical modes (Eq. 3a) should be chosen in such a way, that the basic physical elements identified by the step (1) are well represented. (3) Perform a heavy mode truncation (compression; cf., Eq. 4a) in a given phase space, in order for a parameterization (i.e., the right-hand side of Eq. 2 to be obtained). (4) Perform an inverse transformation (decompression; cf., Eq. 4b) from a phase space to a physical space, in order to obtain downscaling.

Probably, the most popular mathematical choice for a mode decomposition would be Fourier. In convection parameterizations, the mass fluxes are used as basic set of modes. The hydrological equivalence would be basins. A more general mathematical framework is provided by the multiresolution analysis (cf., Mallat, 1998), which provides a basis for wavelet.

The basic set of modes must be chosen in such a way, that it fits well the geometrical structures of the subgrid-scale processes in concern. A glance of any three-dimensional visualizations of a typical atmospheric convective system shows that it consists of ensembles of spatially-isolated coherent structures such as cumulus convective towers (historical "plumes"), convective downdrafts, stratiform cloud ascends, mesoscale downdrafts. Wavelet is a natural choice for the decomposition, because it is exactly developed for a purpose of efficiently representing spatially-isolated coherent structures. Yano et al. (2004) demonstrate that the wavelet can easily compress atmospheric convective systems down to a level of $1 \%$ without substantially losing their overall structure. For this reason, Yano et al. (2005) propose to develop a general SSR based on wavelet.

In this manner, the multiresolution analysis provides a mathematical basis for developing parameterization and downscaling as compression and decompression. A subsequent investigation by the author, however, reveals practical difficulties in developing a wavelet-based SSR. The main difficulty is due to the fact that various physical processes, such as microphysics, must be evaluated in the physical space. Thus, an efficient forward and inverse transformation scheme is required, but that turns out to be rather awkward to develop. A compressed representation can be integrated in time more efficiently, if all the calculations are simply performed in the physical space without any transform into a phase space. This leads to an idea of a segmentally-constant approximation (SCA), which is to be documented in the next section.

\section{A compressed subgrid-scale representation (SSR) under segmentally-constant approximation (SCA)}

A new type of compressed subgrid-scale representation (SSR), called a nonhydrostatic anelastic model (NAM) with segmentally-constant approximation (SCA), or NAMSCA, is developed under the principle discussed in the previous section. For this purpose, we take the mass flux, adopted by the majority of the current convection parameterizations, as a basic set of modes.

\subsection{A single-plume model}

The "mass flux" is a mathematical idealization for representing an ensemble of convective plumes. These spatiallyisolated coherent structures discussed in the previous section may also be interpreted as a generalization of plumes. Each convective plume is approximated by a horizontally homogeneous segment, which is called the mass flux. As a result, each horizontal plane of the system is approximated by pieces of homogeneous segments, named the segmentally constant approximation (SCA).

The simplest configuration under SCA is to place a single convective plume into an otherwise horizontallyhomogeneous grid box domain. The configuration corresponds to the so-called bulk mass flux formulation, an approach that is taken by the majority of mass-flux convection parameterizations. Current standard bulk mass-flux parameterizations are constructed under various additional hypotheses, approximations, as well as closures, but we can construct a prototype model by simply imposing a geometry of a single convective plume. 
For demonstrative purposes, here we take a twodimensional domain (a vertical section) periodic in the horizontal direction. Within this domain, we place a single convective plume, or a updraft, represented by a top-hat profile.

In other words, all the physical variables are approximated by a constant value, i.e., $w_{c}, \theta_{c}, \varphi_{c}$ for the convective plume (updraft), where $w$ and $\theta$ are the vertical velocity and the potential temperature, respectively. A subscript, $c$ is added to indicate a convection component. We also assume that outside the plume segment all the variables are also approximated by constant environmental values i.e., $w_{\mathrm{e}}, \theta_{e}, \varphi_{e}$, where a subscript, $e$ indicates environment. Thus,

$\varphi(x, y, z, t)=\left\{\begin{array}{l}\varphi_{c}(z, t) \text { if } x_{1} \leq x \leq x_{2} \\ \varphi_{e}(z, t) \text { otherwise }\end{array}\right.$

where we assume that a plume occupies a range $x_{1} \leq x \leq x_{2}$ at every height, $z$ of the system.

The governing equation for the plume element $\varphi_{c}(z, t)$ is obtained, as a standard procedure of finite-volume methods, by integrating the original full system (Eq. 1) over a plume segment $\left[x_{1}, x_{2}\right]$ :

$$
\frac{\partial}{\partial t} \sigma_{c} \varphi_{c}+\frac{1}{L}\left(u_{2} \varphi_{2}-u_{1} \varphi_{1}\right)+\frac{1}{\rho} \frac{\partial}{\partial_{z}} \rho \sigma_{c} w_{c} \varphi_{c}=\sigma_{c} F_{c}
$$

where $\sigma_{c}=\left(x_{2}-x_{1}\right) / L$ is a fractional area occupied by the plume, $u_{j}$ and $\varphi_{j}$ with $j=1,2$ are the values at the segment interfaces $x=x_{j}(j=1,2)$ and $F_{c}$ is the forcing averaged over the plume segment. Here, $L$ is a horizontal domain size. An equivalent equation for the environmental segment is also readily obtained.

The system is self-contained, once the density, $\rho$ is prescribed as a fixed vertical profile (a standard approximation used in the anelastic system), the horizontal velocity, $u$ is diagnosed from the vertical velocity, $w_{*}(*=c, e)$ by the mass continuity (note: as a result, $u$ is segmentally linear in the horizontal direction), and the pressure field, $p$ is diagnosed by inverting the Poisson equation. Segment interface values are defined by the upstream approximation. Refer to Yano et al. (2008) for details. ${ }^{3}$

An animation for the evolution of a single convective plume under this configuration is available at ftp://cnrm-ftp. meteo.fr/pub-moana/yano/adgeo/single_plume.ppt:

the plume is initialized with a potential-temperature anomaly of $2 \mathrm{~K}$ at the lowest $2.5 \mathrm{~km}$ of the $10 \mathrm{~km}$ - deep domain. The horizontal domain size is $256 \mathrm{~km}$. For simplicity, the background potential-temperature is set zero, so that no gravity wave would be generated. The animation shows by the evolution of the potential temperature anomaly that the initial thermal plume induces an upward motion by a positive buoyancy, which in turn, lifts the thermal anomaly with time. The plume event ends by the thermal anomaly hitting the top wall.

\footnotetext{
${ }^{3}$ The code and a documentation for the following demonstration is available at ftp://cnrm-ftp.meteo.fr/pub-moana/yano/crm-sca/no_ activation_dry_0/.
}

A virtually realistic plume evolution is simulated by a rather simple numerical configuration.

\subsection{Multiple-plume models}

The single-plume model well demonstrates the basic idea of NAM-SCA. However, the model can only simulate a single plume event. An event is simply transient, and once an event is over, nothing happens in the system any more, unless a plume is re-initialized. Such a re-initialization procedure is far from trivial, although certain artificial conditions may be invented. An easier approach is to add more plumes (i.e., segmentally constant segments) into the model domain, so that the multiple plumes would continuously evolve in time without artificial re-initializations. However, as more plumes (constant segments) are added, the model becomes less compressed. In order to maintain a high compression of the model, constant segments must also be removed as soon as a plume event is over. New constant segments must be added only when a new plume begins to glow upwards. In this manner, we end up at a highly-flexible adaptive mesh refined scheme.

The implementation simply follows the above physical principle: we retain a full resolution (as the resolution used for solving the pressure Poisson problem) for the first few layers of the model throughout the simulation. The model is initialized with a random potential-temperature perturbation in the first two layers. These initial noises induce plumes with time. Once a plume is triggered, constant segments are added upward with time ahead of an upward evolution of the plume. As a plume event is over, these constant segments are simply removed. For the details refer to Yano et al. (2010).

Two examples of animations are provided as supplementary materials. ${ }^{4}$ The first case (ftp: //cnrm-ftp.meteo.fr/pub-moana/yano/adgeo/bl_plumes.ppt) simulates dry boundary-layer turbulent convection. It corresponds to the case $24 \mathrm{~F}$ given by Ayotte et al. (1996): the case simulates a well-mixed dry convective boundary layer of about $1 \mathrm{~km}$ depth. A horizontal domain is $3.2 \mathrm{~km}$. An artificially strong inversion is placed at the top of the boundary layer in order to ensure its stability (cf., Fig. 5 of Yano et al., 2010). The upper frame of the animation shows the vertical velocity along with the boundaries of constant segments indicated by vertical bars. The lower frame shows the potential temperature as a deviation from a horizontal average. After a transient initial period, a few thermal plumes (each of them survives for 10-20 min), constantly occupy the domain. The animation shows that the developed time-dependent mesh-refined scheme only puts meshes where plumes are active, and these segments are removed when a plume episode is over.

\footnotetext{
${ }^{4}$ The codes used for these two demonstrations are available at ftp://cnrm-ftp.meteo.fr/pub-moana/yano/crm-sca/with_activation_ayotte/, ftp://cnrm-ftp.meteo.fr/pub-moana/yano/crm-sca/with_activation_moist_1/, respectively.
} 
The second case (ftp://cnrm-ftp.meteo.fr/pub-moana/ yano/adgeo/gate.ppt) simulates a shear-driven organized tropical squall-line system. The case is taken from Jung and Arakawa (2005), in which an idealized large-scale forcing is defined based on a GATE (Global Atmospheric Research Program - GARP - Atlantic Tropical Experiment) PhaseIII mean observation. The horizontal domain is $512 \mathrm{~km}$. In this case, the moisture is introduced into the system, and the cloud water and precipitation are handled based on a methodology developed by Kessler (1965). The animations of the second case show from the top frame to bottom: the vertical velocity $(\mathrm{m} / \mathrm{sec})$, the potential temperature (as a deviation from a horizontal mean, K), the water-vapor mixing ratio $(\mathrm{g} / \mathrm{kg})$, the cloud-water mixing ratio $(\mathrm{g} / \mathrm{kg})$, and the precipitating-water mixing ratio $(\mathrm{g} / \mathrm{kg})$.

A westward-propagating squall-line system is successfully simulated by placing intensive mesh refinements only over convective regions. The dense mesh region also shifts, as the squall-line system propagates with time. Outside the convective regions, almost no mesh refinement is applied, and the model runs successfully only with the eight constant segments posed homogeneously over the whole domain as the minimum model resolution.

\section{Conclusions}

The present paper emphasizes that the downscaling and parameterization constitute two parts of a single problem of the subgrid-scale representation (SSR). Unfortunately, the current downscaling and parameterization schemes are not constructed in such manner. Some reasons for this limitation are discussed.

A systematic, self-consistent methodology for developing both downscaling and parameterization together as a pair is, then proposed, based on mode decomposition (Yano et al., 2005). Under this framework, compression of the original full system for the subgrid-scale processes (e.g., a regional model, a cloud-resolving model) leads to a parameterization, because it provides a compact description of subgrid-scale processes. By decompressing this compressed information, a spatial information on the subgrid-scale processes is recovered, thus a downscaling is achieved subsequently. A mathematical theory called multiresolution analysis (Mallat, 1998) provides a conceptual framework for this purpose.

As an example of this compression-decompression approach, the nonhydrostatic anelastic model (NAM) with segmentally-constant approximation (SCA), or NAM-SCA is introduced. NAM-SCA is based on the idea of decomposing a subgrid-scale atmospheric system into an ensemble of convective plumes. Each plume is assumed to take a horizontally-homogeneous distribution of physical variables over a finite segment at each vertical level, thus the concept of SCA follows. Mathematically, the method is an application of a spirit of the multiresolution analysis within a context of a finite volume approach. Physically, NAM-SCA can be considered as a prototype of the mass-flux convection parameterization (Arakawa and Schubert, 1974), but without closure assumptions.

The idea of NAM-SCA is demonstrated for the simplest case with a single plume and more complex situations with multiple plumes. In the latter case, the segmentally-constant segments are activated and deactivated with time by following the evolution of plumes.

Acknowledgements. The present paper is based on a material presented at the 10th Plinius Conference held in Cyprus, September 2008. The author would like to thank E. Foufoula-Georgiou for providing him an occasion for a presentation. Critical readings by Ioannis Pytharoulis and anonymous reviewer as well as an editorial work by Artemis Orphanou are also acknowledged.

Edited by: S. Michaelides, K. Nicolaides, and A. Orphanou Reviewed by: I. Pytharoulis and one anonymous referee

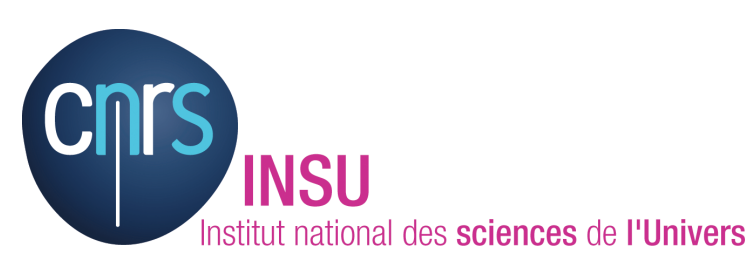

The publication of this article is financed by CNRS-INSU.

\section{References}

Arakawa, A.: The cumulus parameterization problem: Past, present, and future, J. Climate, 17, 2493-2525, 2004.

Arakawa, A. and Schubert, W. H.: Interaction of a cumulus cloud ensemble with the large-scale environment, part I, J. Atmos. Sci., 31, 674-701, 1974.

Ayotte, K. W., Sullivan, P. P., Andrien, A., Doney, S. C., Holtslag, A. A. M., Large, W. G., McWilliams, J. C., Moeng, C.-H., Otte, M. J., Tribbia, J. J., Wyngaard, J. C., An evaluation of neutral and convective planetary boundary-layer parameterizations relative to large eddy simulations, Bound.-Lay. Meteorol., 79, 131$175,1996$.

Foufoula-Georgiou, E.: On scaling theories of space-time rainfall: Some recent results and open problems, in: Stochastic Methods in Hydrology: Rain, Landforms and Floods, edited by: Barndorff-Nielsen, O. E., Gupta, V. K., Perez-Abreu, V., and Waymire, E. C., Vol. 7 in the Advanced Series on Statistical Sciences and Applied Probability, Word Scientific, 1997.

Grabowski, W. W. and Smolarkiewicz, P. K.: CRCP: A cloud resolving convection parameterization for modeling the tropical convective atmosphere, Physica D, 133, 171-178, 1999.

Jung, J.-H., and Arakawa, A.: Preliminary tests of multiscale modeling with a two-dimensional framework: Sensitivity to coupling methods, J. Atmos. Sci., 133, 649-662, 2005.

Kessler III, E.: Microphysical parameters in relation to tropical cloud and precipitation distributions and their modifications, Geofisica International, 5(3), July 1975, 79-88, 1965. 
Marani, M. and Zanetti, S.: Downscaling rainfall temporal variability, Water Resour. Res., 43, W09415, doi:10.1029/2006WR00505, 2007.

Mallat, S.: A Wavelet Tour of Signal Processing, 2nd edition, Academic Press, 637 pp., 1998.

Yano, J.-I., Bechtold, P., Redelsperger, J.-L., and Guichard, F.: Wavelet-Compressed Representation of deep moist convection, Mon. Weather Rev., 132, 1472-1486, 2004.

Yano, J.-I., Redelsperger, J.-L., Guichard, F., and Bechtold, P.: Mode decomposition as a methodology for developing convective-scale representations in global models, Q. J. Roy. Meteorol. Soc., 131, 2313-2336, 2005.
Yano, J.-I., Bernardet, P., Benard, P., and Lahellec, A.: NAM-SCA: Nonhydrostatic Anelastic Model under Segmentally-Constant Approximation: I. Dry Formulation and A Phase-Space Investigation of the Single Plume Dynamics, ftp://cnrm-ftp.meteo. fr/pub-moana/yano/dry_case/ms.pdf, last access: 11 September 2008, manuscript rejected by Mon. Weather Rev., 2008.

Yano, J.-I., Benard, P., Couvreux, F., and Lahellec, A.: NAM-SCA: Nonhydrostatic Anelastic Model under Segmentally-Constant Approximation, Mon. Weather Rev., 138, 1957-1974, 2010. 